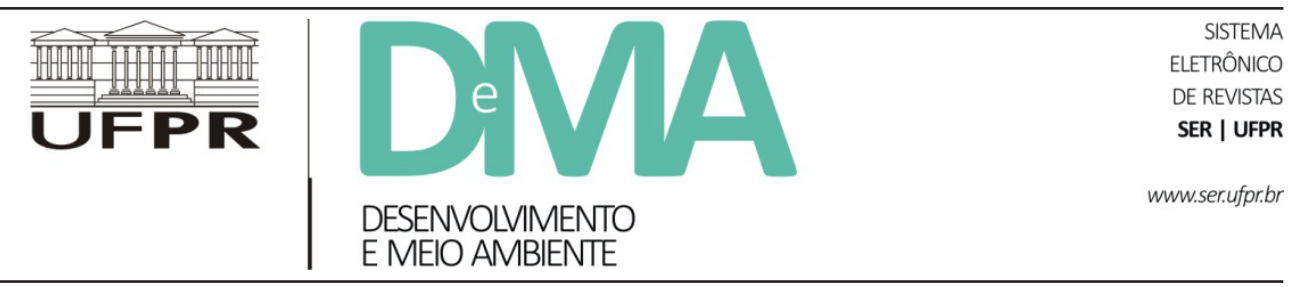

\title{
Etnoentomologia em comunidade rural do cerrado piauiense
}

\section{Ethnoentomology in a Rural Community from Piauí State Cerrado}

\author{
Brunna Laryelle Silva BOMFIM ${ }^{1 *}$, Irineu Campêlo da FONSECA FILHO ${ }^{2}$, Juliana Cardozo de FARIAS 3 , \\ Solange Maria de FRANÇA ${ }^{4}$, Roseli Farias Melo de BARROS ${ }^{5}$, Paulo Roberto Ramalho SILVA ${ }^{5}$ \\ ${ }^{1}$ Instituto Federal do Piauí (IFPI), Uruçuí, PI, Brasil. \\ ${ }^{2}$ Instituto Federal do Piauí (IFPI), Angical do Piauí, PI, Brasil. \\ ${ }^{3}$ Secretaria Municipal de Educação, Santana, MA, Brasil. \\ ${ }^{4}$ Universidade Federal Rural de Pernambuco (UFRPE), Recife, PE, Brasil. \\ ${ }^{5}$ Universidade Federal do Piauí (UFPI), Teresina, PI, Brasil. \\ *E-mail de contato: brunnalaryelle@yahoo.com.br
}

Artigo recebido em 31 de dezembro de 2015, versão final aceita em 30 de setembro de 2016.

RESUMO: Os insetos apresentam extrema importância para a natureza, porém são muitas vezes percebidos de forma negativa. Tendo isso em vista, objetivou-se avaliar a percepção e o uso deles pela população da comunidade Sussuapara, em Nazaré do Piauí, no estado do Piauí, Brasil. Foram entrevistados 89 moradores, por meio do emprego de formulários semiestruturados. Para a coleta dos insetos, foram deixados potes com os entrevistados. A análise dos dados se deu de forma quantitativa. O nível de fidelidade (FL), a prioridade de ordenamento (ROP) e o valor de importância $\left(\mathrm{IV}_{\mathrm{s}}\right.$ ) foram calculados para as espécies com uso medicinal e espécies citadas como pragas. Para os entrevistados, o etnodomínio "inseto" é composto de 49 organismos, constituídos por animais nem sempre classificados sistematicamente na classe Insecta. $O$ uso de insetos se restringiu a categoria medicinal, sendo apontado o mel de abelha (produto de inseto) em $93 \%$ das entrevistas ( $\mathrm{FL}=0,93, \mathrm{ROP}=0,9$ ). Na categoria "alimentos", o mel é o único produto utilizado. A comunidade reconhece como pragas insetos que causam apenas danos na lavoura, destacando a lagarta (Lepidoptera) $\left(\mathrm{IV}_{\mathrm{s}}=0,9\right)$ e a formiga (Hymenoptera) $\left(\mathrm{IV}_{\mathrm{s}}=0,7\right)$ como principais pragas agrícolas e utilizando exclusivamente inseticidas sintéticos no seu controle. A percepção negativa a respeitos dos insetos aliada à ausência do conhecimento, pela comunidade, acerca da importância deles na natureza fortalece ações agressivas contra tais animais.

Palavras-chave: uso de insetos; etnobiologia; conhecimento.

ABSTRACT: Insects are extremely important for nature, however, they are often perceived negatively. Therefore, this study aims at evaluating the perception and use of the insect population in Sussuapara community, Piauí Nazareth/ PI, Brazil. A total of 89 residents were interviewed, using semi-structured forms. In order to collect the insects, bowls were left to the respondents. The analysis of the data was done quantitatively. The Fidelity Level (FL), ranking order priority (ROP) and importance value (IV) were calculated for the species with medicinal use and 
species cited as pests. According to interviewees, the ethno domain "insect" is composed of 49 organizations consisting of animals not always systematically classified in Class Insecta. The use of insects was restricted to medical category, wherein the honey bee (insect product) was appointed in $93 \%$ of the interviews ( $\mathrm{FL}=0.93$, ROP $=0.9$ ). In the category "food", honey is the only product used. The community recognizes as plagues only insects that cause damage in their farming, highlighting the caterpillar (Lepidoptera) (IVs $=0.9$ ) and ants (Hymenoptera) $(\mathrm{IVs}=0.7)$ as the main agricultural pests, and using only synthetic insecticides in their control. The negative perception of the insects combined with the lack of knowledge about the importance of insects in nature among the community strengthens aggressive actions against them.

Keywords: use of insects; ethnobiology; knowledge.

\section{Introdução}

Os insetos estão entre os grupos de animais com os quais o homem mantém estrita relação. Eles estão presentes na alimentação, em práticas médicas, superstições, crenças, brincadeiras, e substâncias produzidas por tais animais são utilizadas, tais como mel, própolis e geleia real (Modro et al., 2009a; Ramandey \& Mastrigt, 2010; Klein, 2012). Tal relação é estudada pela etnoentomologia, definida por Petiza et al. (2013a, p. 323) como "um ramo da Etnociência que busca compreender a maneira como diversas culturas percebem, identificam, classificam, nomeiam, utilizam e conhecem o que entendem por 'inseto' em suas línguas".

Trabalhos etnoentomológicos são desenvolvidos em diversos países ao redor do mundo, dentre os quais se destacam a Índia e a Tailândia, os quais apresentam o uso de insetos principalmente na alimentação e na medicina (Mahawar \& Jaroli, 2008; Durst \& Shono, 2010; Meyer-Rochow, 2010; Meyer-Rochow \& Chakravorty, 2013). O uso de insetos na alimentação, por exemplo, é apresentado como potencial fonte de renda para diversas comunidades, pois, quando comparada à produção de outros grupos animais para fins alimentícios, como mamíferos ou aves, a criação de insetos se torna muito mais econômica (Durst \& Shono, 2010). Já em comunidades indianas, os insetos se destacam como um dos principais invertebrados utilizados para fins terapêuticos (Mahawar \& Jaroli, 2008).

No Brasil, os estudos etnozoológicos tiveram início na década de 1930, tendo sido publicados, até 2011, aproximadamente 500 trabalhos. Destes, cerca de $13 \%$ são estudos etnoentomológicos (Alves \& Souto, 2011). O crescimento no número de pesquisas etnoentomológicas se deve à facilidade no acesso a informações sobre insetos, já que o uso de tais animais não implica problemas legais, diferentemente do que acontece, por exemplo, quando o tema abordado é caça. $\mathrm{O}$ fato de essa prática ser proibida no Brasil dificulta o acesso do pesquisador às informações sobre o uso de tais recursos (Alves \& Souto, 2011).

Dentre os temas mais abordados nas pesquisas etnoentomológicas brasileiras está a percepção que comunidades ou grupos têm acerca dos insetos, tais como os conduzidos por Ferreira et al. (2010) com os índios kaiabi, os quais apresentaram seus conhecimentos a respeito das abelhas nativas sem ferrão, bem como os usos dos produtos de tais animais em sua cultura. Outro estudo, feito com alunos de ensino fundamental no município de Uruará (PA), a respeito da percepção dos alunos sobre insetos antes e após aulas práticas, demonstrou que a percepção acerca dos insetos é geralmente negativa, com sentimentos de repúdio, nojo, além do medo trazido por insetos nocivos e pragas. No entanto, 
uma pequena parcela dos entrevistados aparentou percepção positiva, apontando a importância dos insetos para a natureza devido à sua ação polinizadora e decompositora, bem como sua importância na alimentação de animais e na produção alimentícia para o homem (Cajaíba \& Silva, 2015). Outro aspecto estudado é o levantamento sobre etnotaxonomia de insetos, como os realizados em comunidades do Amazonas, bem como da Bahia e da Paraíba (Costa Neto \& Pacheco, 2004; Petiza et al., 2013b).

No Piauí, estudos etnoentomológicos versam sobre sua utilização e a de seus produtos na alimentação, bem como na medicina local, além da classificação do etnodomínio "insetos" por diversas comunidades (Souza \& Lima, 2014; Almeida et al., 2015). Em pesquisa etnoentomológica realizada em uma comunidade na serra de Passa Tempo, município de Campo Maior (PI), foram apresentadas as percepções que os moradores locais tinham sobre insetos (Almeida et al., 2015). Já na capital, Teresina, foram levantados o conhecimento e o uso de insetos em hortas comunitárias e nos mercados públicos do município (Souza \& Lima, 2014).

Pressupondo que os indivíduos de Sussuapara possuem conhecimento sobre a forma de vida dos insetos e suas relações com o meio ambiente e que muitos dos animais citados por esses indivíduos como "insetos" são, na realidade, classificados pela taxonomia lineana em outras categorias distintas da classe Insecta, objetivou-se apresentar a percepção e o uso dos insetos pela população da comunidade Sussuapara, no município de Nazaré do Piauí (PI).

\section{Metodologia}

\section{1. Área de estudo}

Para a realização deste estudo foi escolhida a comunidade de Sussuapara $\left(07^{\circ} 01^{\prime} 02^{\prime}\right.$ 'S e
4241'54,6”W), localizada no município de Nazaré do Piauí, estado do Piauí, Nordeste do Brasil, o qual foi elevado à categoria de cidade em 1955 e está inserido na microrregião de Floriano, apresentando como limites os municípios de Francisco Ayres e Floriano ao norte; ao sul e a oeste, Floriano; e, a leste, Oeiras, São Francisco do Piauí, São José do Peixe e Cajazeiras do Piauí (Aguiar \& Gomes, 2004). Possui área de $1.311,565 \mathrm{~km}^{2}$ e se localiza na área delimitada como Polígono das Secas, apresentando clima tropical semiárido quente, com período seco de sete a oito meses. As temperaturas médias variam entre $26^{\circ} \mathrm{C}$ e $37^{\circ} \mathrm{C}$ e há vegetação do tipo cerradão e campo cerrado (CEPRO, 2010).

A comunidade Sussuapara teve origem no século XIX, quando famílias de outras comunidades chegaram à região. Atualmente conta com 51 famílias e cerca de 180 pessoas, de acordo com informações obtidas com o agente de saúde local. Em relação à faixa etária dos moradores da comunidade, $27 \%$ possuem idade entre 0 e 17 anos, $13 \%$ estão na faixa de 18 a 24 anos, $50 \%$ têm entre 25 e 59 anos e apenas $10 \%$ dos moradores possuem idade acima de 60 anos. A renda mensal da maioria das famílias (55\%) está entre um e três salários mínimos, $43 \%$ das famílias possuem renda mensal de até um salário mínimo e $2 \%$ informaram renda entre três e seis salários mínimos. A agricultura de subsistência é a principal atividade econômica da comunidade, servindo de base para o sustento da maioria das famílias, bem como complementação de renda das demais famílias. Os produtos cultivados pelos moradores são arroz (Oryza sativa L.), milho (Zea mays L.), feijão [Vigna unguiculata (L.) Walp, Phaseolus vulgaris L., Phaseolus lunatus L.], cana-de-açúcar (Saccharum officinarum L.) e mandioca (Manihot esculenta Crantz). Algumas famílias cultivam hortaliças como coentro ( $\mathrm{Co}$ riandrum sativum L.), cebolinha (Allium fistulosum 
L.), tomate (Solanum lycopersicum L.), pimentão (Capsicum annuum L.), alface (Lactuca sativa L.), dentre outras. A pecuária é a segunda atividade econômica mais importante da localidade, sendo representada pela criação de pequenos rebanhos bovinos, suínos, caprinos, ovinos e aves.

\subsection{Coleta e análise dos dados}

A pesquisa utilizou metodologia exploratória, descritiva e interpretativa e abordagem quantitativa com organização de lista com as etnoespécies citadas, seguidas do nome científico de cada uma (Huberman \& Miles, 1991; Mucchielli, 1996; Rocha et al., 2008).

Seguindo as normas estabelecidas para pesquisas com seres humanos, esta pesquisa foi aprovada pelo Comitê de Ética em Pesquisa (CEP) da Universidade Federal do Piauí (UFPI) sob o Certificado de Apresentação para Apreciação Ética (CAAE) 36813514.6.0000.5214. O Termo de Consentimento Livre e Esclarecido (TCLE) foi lido e explicado antes de cada entrevista, sendo requerida a assinatura de cada entrevistado. Foi realizada palestra com a comunidade a fim de tornar conhecido o trabalho que seria desenvolvido.

Para a coleta de dados, foram realizadas entrevistas com a população a fim de saber quais insetos são percebidos na comunidade e como estes são classificados com bases morfológica, comportamental e ecológica pelos moradores. Devido ao reduzido tamanho da comunidade, o estudo foi realizado com todas as famílias, como utilizado por Begossi et al. (2009), sendo escolhidos, quando presentes, até três componentes de cada núcleo familiar (o pai, a mãe e um filho maior de 18 anos). A entrevista foi realizada com aplicação de formulário semiestruturado. Inicialmente foram levantados os dados socioeconômicos de cada família entrevistada e posteriormente foi realizada metodologia de estímulos visuais, conforme utilizada por Ulysséa et al. (2010). Tal procedimento é realizado a partir da apresentação de imagens ou de representantes de espécies coletadas, de forma a reconhecer a nomenclatura adotada pela comunidade (Wester \& Yongvanit, 2006). Neste estudo, adotou-se apenas o uso de imagens. Foram apresentadas figuras de espécies de artrópodes, anfíbios, répteis e mamíferos, de forma que os entrevistados puderam apontar quais eram considerados insetos e o nome utilizado para denominar cada representante visualizado.

Foram realizadas 89 entrevistas, com representantes de 46 famílias, dentre os quais 47 eram mulheres (53\%) e 42 eram homens (47\%), com idades entre 18 e 82 anos. A diferença entre o total de famílias e indivíduos residentes e o total de entrevistados se deu pelo fato de que muitos moradores da localidade também possuem residência em outros municípios e não estavam presentes no momento da pesquisa, enquanto outros não foram encontrados na comunidade em nenhuma das visitas realizadas.

Para coleta dos "insetos" citados, foram deixados potes com alguns entrevistados, para que estes coletassem, quando possível, aqueles citados durante a entrevista. Houve orientação quanto à forma de coleta (Costa Neto, 2003). Os potes foram deixados em cinco residências localizadas em pontos estratégicos da comunidade, recolhidos após uma semana e encaminhados para o Laboratório de Fitossanidade da UFPI, onde se procedeu à identificação taxonômica. Os entrevistados coletaram representantes de besouros (Coleoptera), abelhas (Apoidea), cupins (Isoptera), borboletas (Lepidoptera) e libélulas (Odonata). A conferência da grafia e do nome dos autores das espécies aqui citadas seguiu o indicado pelo ITIS (2015). 
Para análise dos dados de uso dos insetos pela população foram utilizados o nível de fidelidade (FL), proposto por Friedman et al. (1986) e utilizado por Ulysséa et al. (2010) - os quais afirmam que o FL apresenta o grau de correspondência entre as respostas dos entrevistados quanto aos usos citados para um animal, sendo $\mathbf{F L}=(\mathbf{I p} / \mathbf{I u}) \times \mathbf{1 0 0} \%$ (onde: $\mathbf{F L}=$ nível de fidelidade; $\mathbf{I p}=$ número de informantes que citaram o uso principal da espécie; $\mathbf{I u}=$ número total de informantes que citaram a espécie para qualquer finalidade) -, e a prioridade de ordenamento (Friedman et al., 1986), sendo ROP $=\mathbf{F L} \mathbf{x} \mathbf{R P}$ (em que: $\mathbf{R O P}=$ prioridade de ordenamento; $\mathbf{F L}=$ nível de fidelidade; $\mathbf{R P}=$ popularidade relativa, calculada pelo número de informantes que citaram um dado inseto, dividido pelo número de informantes que citaram o inseto mais citado). O valor de importância $\left(\mathrm{IV}_{\mathrm{s}}\right.$ ) foi calculado para as espécies citadas na categoria de pragas, com o objetivo de identificar as espécies de maior importância local. Foi determinado pela fórmula $\mathbf{I V}=\mathbf{n}_{\mathbf{s}} / \mathbf{n}$, onde $\mathbf{n}_{\text {is }}$ é o número de informantes que consideraram a espécie "s" mais importante e $\mathbf{n}$, o número total de informantes (Byg \& Balslev, 2001).

\section{Resultados e discussão}

\subsection{Concepções sobre insetos}

A comunidade Sussuapara reconhece 49 organismos como insetos, dos quais 31 são classificados sistematicamente na classe Insecta (Tabela 1) e 18 são classificados em oito táxons diferentes, dentre os quais se destacam a lacraia/escorpião (Scorpiones), com 79\% das citações, seguida pela cobra (Squamata) e por aranhas (Araneae), com $62 \%$ e $55 \%$ respectivamente (Tabela 2 ). Classificar como insetos animais pertencentes a outras classes é comum entre os diversos povos ao redor do mundo. Trabalhos etnoentomológicos apontam que as populações classificam, juntamente com os insetos, um número considerável de espécies de outros grupos (Petiza et al., 2013a; 2013b). Indivíduos que residem em comunidades rurais, geralmente, associam os insetos com animais pequenos, que causam nojo ou que não têm nenhuma função aparente. De acordo com Montenegro et al. (2014), a etnocategoria inseto é construída no cotidiano das comunidades influenciada pela cultura local. Dessa forma, os artrópodes em geral, assim como outros animais, são classificados como insetos.

Para os entrevistados, insetos são animais pequenos $(81 \%)$, mordedores $(74 \%)$, barulhentos (66\%) e que podem voar (23\%). Alguns são bonitos $(33 \%)$. Houve entrevistados, porém, que não viram características agradáveis nos insetos, como percebido nas palavras de alguns moradores: "tudo é feio" (Sra. L., 57 anos), "é mau” (Sr. F., 53 anos) e "pra fazer o mal é bom" (Sr. R., 44 anos) (figura 1). O que detectamos em relação aos insetos na comunidade estudada corrobora o referido por Modro et al. (2009b) em trabalho realizado com docentes e discentes no interior de Mato Grosso, onde os insetos também foram caracterizados como animais pequenos e perigosos. A noção de periculosidade a respeito de tais animais também aparece em outros trabalhos etnoentomológicos, como o de Costa Neto \& Pacheco (2004) e Almeida Neto et al. (2015), os quais registram a caracterização de insetos como animais perigosos entre os indivíduos entrevistados. O uso de adjetivos como "bonito", "feio", "agradável", "bom" ou "mau" é comumente associado aos insetos e verificado em vários trabalhos (Lopes et al., 2014; Souza et al., 2014). Isso apresenta a forma como as pessoas percebem tais animais. Por meio das respostas obtidas durante as entrevistas, notou-se que as borboletas (Lepidoptera) e libélu- 
TABELA 1 - Animais citados pela comunidade Sussuapara, em Nazaré do Piauí (PI), e classificados sistematicamente na classe Insecta

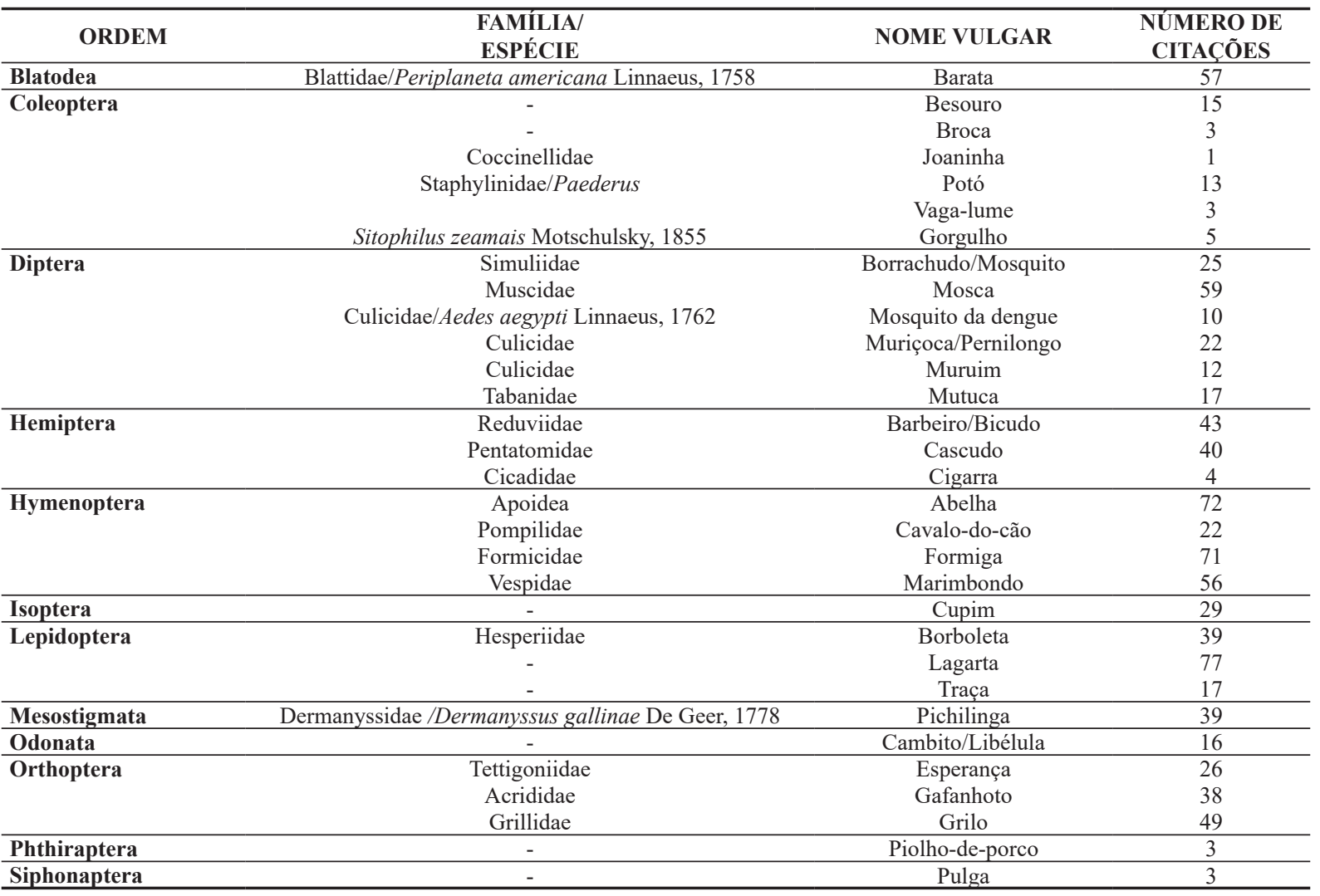

TABELA 2 - Animais de outros táxons classificados como insetos pela comunidade Sussuapara, em Nazaré do Piauí (PI)

\begin{tabular}{|c|c|c|c|}
\hline CLASSE & ORDEM/FAMÍLIA & NOME VULGAR & $\begin{array}{l}\text { NÚMERO DE } \\
\text { CITAÇÕES }\end{array}$ \\
\hline Annelida & Haplotaxida & Minhoca & 6 \\
\hline Amphibia & Bufonidae & Sapo & 19 \\
\hline \multirow{2}{*}{ Arachnida } & Scorpiones & Escorpião/Lacraia & 70 \\
\hline & Ixodida & Carrapato & 6 \\
\hline Chilopoda & Scolopendromorpha & Piolho-de-cobra & 22 \\
\hline Diplopoda & Julidae & Gangoji & 13 \\
\hline \multirow[t]{4}{*}{ Mammalia } & Didelphidae & Gambá/Mucura & 2 \\
\hline & Chiroptera & Morcego & 30 \\
\hline & Canidae & Raposa & 3 \\
\hline & Muridae & Rato & 44 \\
\hline Repitilia & Teiidae & Tiú & 1 \\
\hline
\end{tabular}


las/cambitos (Odonata) muitas vezes são correlacionados a momentos lúdicos na infância e geralmente caracterizados como "bonitos" ou "engraçados", sendo percebidos, em geral, de forma positiva pelos entrevistados. As abelhas (Hymenoptera), embora tidas como perigosas devido aos acidentes que podem causar, são percebidas de forma positiva por alguns moradores que reconhecem a importância do mel produzido por elas. Já aqueles que tiveram alguma experiência ruim relacionada aos insetos, sobretudo os "peçonhentos", ou que se incomodam com a presença deles em suas casas, por exemplo, tendem a percebê-los de forma negativa, como afirmado nas palavras a seguir: "é feio e nojento" (Sra. A., 38 anos) e "bicho perigoso, incomoda e morde" (Sra. N. 39 anos). Segundo Kellert (1993), a mudança na percepção acerca dos insetos é possível. Em trabalho realizado pelo autor com representantes de diversos grupos de pessoas no estado de Connecticut, nos Estados Unidos, os agricultores entrevistados, por exemplo, geralmente, mudam suas concepções acerca de insetos quando veem os benefícios que estes podem trazer para a natureza. Notou-se ainda que a forma como os insetos são percebidos também é influenciada pelo grau acadêmico dos entrevistados, já que os indivíduos com maior escolaridade ou que ainda estavam na escola apresentaram respostas mais semelhantes ao conhecimento acadêmico, como classificação de espécies como insetos e funções deles na natureza.

Quanto à importância dos insetos, $40 \%$ dos informantes afirmaram não saber se existe ou não alguma importância deles para a natureza, outros $40 \%$ disseram que os insetos "não servem para nada" e 4\% informaram que os insetos têm alguma importância no meio ambiente, porém não souberam citar exemplos. A comunidade Sussuapara possui nível de escolaridade baixo. Dentre os entrevistados, apenas duas pessoas informaram possuir curso de graduação, enquanto a maioria afirmou estar fora da sala de aula há muitos anos, possuindo principalmente o ensino fundamental incompleto. Tal fato pode contribuir para a ausência de indicações dos informantes sobre a importância e a função

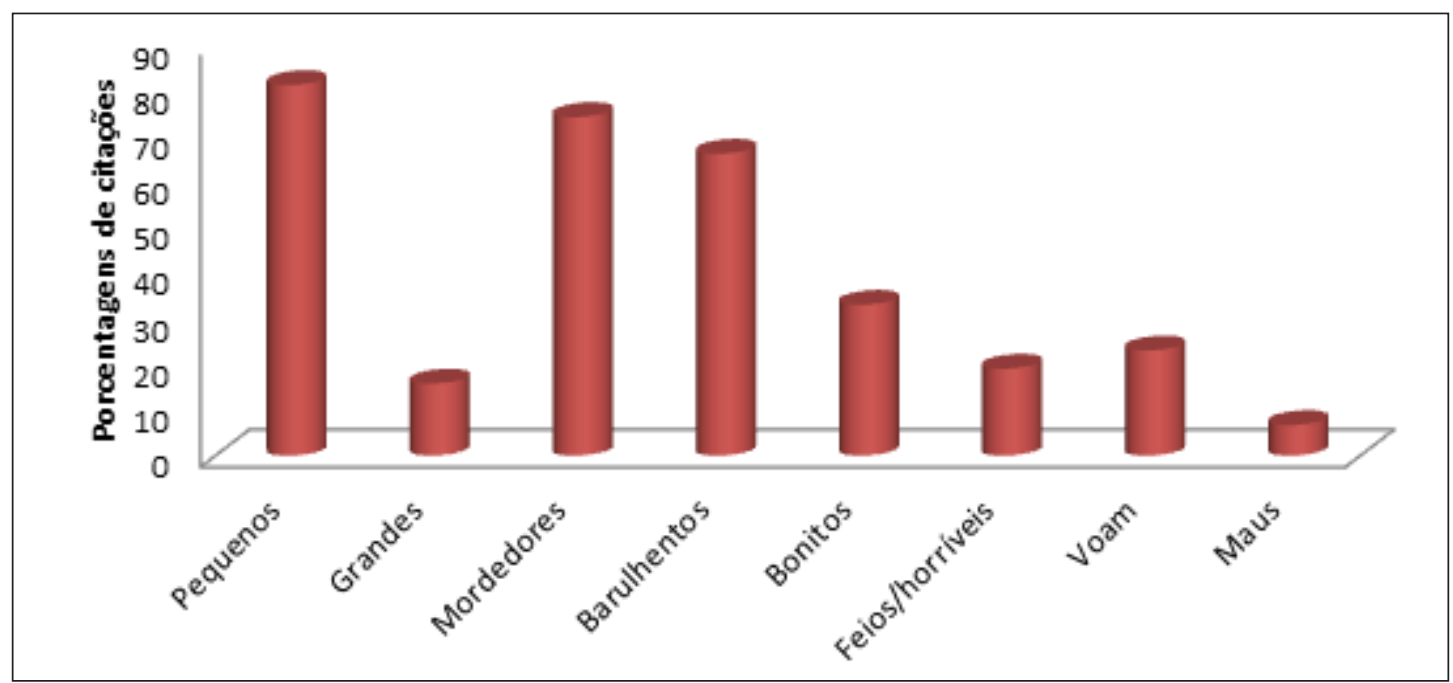

FIGURA 1 - Características gerais dos insetos segundo os moradores da comunidade Sussuapara, em Nazaré do Piauí (PI). 
dos insetos no meio ambiente, influenciando uma concepção errônea sobre esse grupo de animais, conceituados como seres sem utilidade, como demonstrado nas palavras de $40 \%$ dos moradores entrevistados, ao citarem a frase: "não servem para nada". A percepção da importância dos insetos foi relatada entre os indivíduos que possuíam algum curso de graduação ou que haviam concluído recentemente o ensino médio, podendo-se perceber a influência do meio acadêmico na percepção de componentes do cotidiano desses informantes. Esse resultado é semelhante ao encontrado por Costa Neto \& Pacheco (2004, p. 84), os quais afirmam que o ensino formal influencia "a maneira como os organismos são percebidos e classificados". Além das características apontadas, grande parte dos entrevistados indicou diversos outros aspectos relacionados aos insetos. O atributo "perigoso" foi o mais citado, apontado por $100 \%$ dos entrevistados, seguido de "praga" (97\%) e "remédio" (94\%) (Figura 2). A noção de periculosidade dos insetos é percebida em diversos trabalhos etnoentomológicos. Em pesquisa realizada no povoado Pedra Branca, no interior da Bahia, por exemplo, os entrevistados caracterizaram diversas espécies de insetos como perigosos (Costa Neto \& Pacheco, 2004). A concepção sobre a periculosidade dos insetos também é apresentada em estudo realizado em uma comunidade da zona urbana de Cuiabá (MT), onde os entrevistados destacaram a capacidade dos insetos em transmitir doenças (Jorge et al., 2014). Cajaiba \& Silva (2015) também apresentam a nocividade como característica atribuída aos insetos por alunos do ensino fundamental de uma comunidade no interior do Pará. Segundo Trindade et al. (2012, p. 48), a nocividade "é o elemento que apresenta maior resistência à mudança, constituindo o componente que induz a predominância do significado pejorativo verificado para os insetos". Dessa forma, é de se esperar que, por conhecer os riscos que alguns desses animais podem trazer ao homem, a maioria das pessoas apresente reações negativas relacionadas aos insetos, além da tendência de estender tal sentimento aos insetos em geral, independentemente de serem mesmo venenosos. Tal fato é confirmado ao se analisar os sentimentos relacionados aos insetos que os entrevistados apontaram. O sentimento de nojo foi o mais assinalado (68\%), seguido por incômodo $(23 \%)$ e medo (4\%), além de os animais serem considerados ofensivos $(6 \%)$. Os relatos a seguir demonstram tais sentimentos: "causa medo ao ver" (Sra. Z., 33 anos), "eles ofendem as pessoas" (Sra. A., 38 anos), "entra em casa para prejudicar" ( $\mathrm{Sr}$. A., 53 anos), "não serve, só para o mal" (Sr. R., 47 anos). O sentimento negativo causado por esses animais na população parece estar atrelado à aparência desagradável de alguns insetos, à capacidade de transmitirem doenças, bem como ao incômodo que trazem quando presentes nas residências dos entrevistados. Tal constatação parte da análise das entrevistas, em que os informantes demonstravam repulsa ao falar desse grupo animal, apresentando tal comportamento ao fazerem afirmações como: "horrível" (Sra. V., 30 anos), "ofende as pessoas" (Sra. A., 38 anos), "pro mal ele é bom" (Sr. R., 44 anos) e "só pra atentar" (Sra. R., 27 anos). Em trabalho realizado em uma comunidade de Cuiabá (MT), a percepção negativa a respeito dos insetos também foi associada a sentimentos de nojo e medo (Jorge et al., 2014). Por despertarem tais sensações nas pessoas, esses animais podem sofrer ações negativas por parte do homem. O medo, por exemplo, pode estimular ações agressivas contra esses animais, como as percebidos nas palavras de uma moradora entrevistada: "bicho que pica tem que matar" (Sra. L., 35 anos). Trabalhos que exploram essa temática geralmente apresentam como resultado tal percepção quanto aos insetos, como detectado 


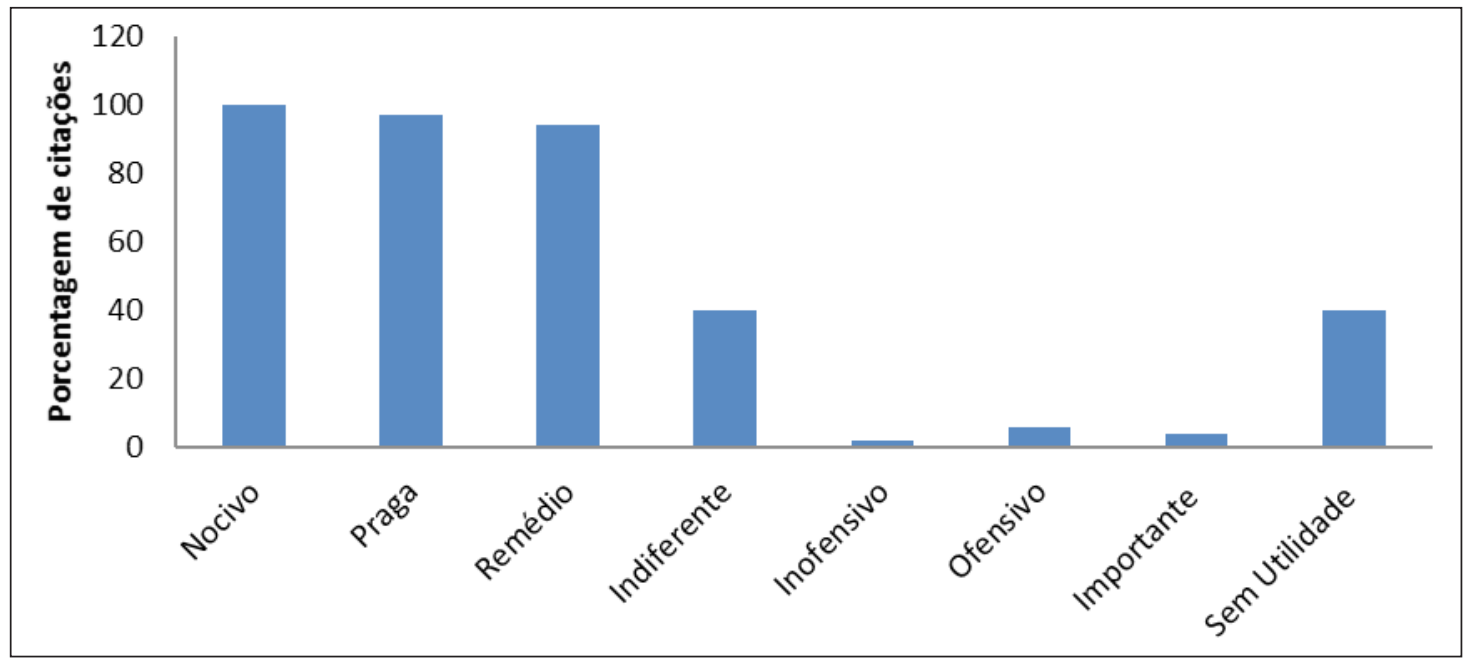

FIGURA 2 - Percepção dos moradores da comunidade Sussuapara, em Nazaré do Piauí (PI), quanto à utilidade dos insetos.

na pesquisa de Almeida Neto et al. (2015, p. 5), em que, por exemplo, grande parte dos insetos citados foi associada com características como "causadores de alguma ofensa, injúria ou prejuízo individual e à coletividade", e em pesquisa realizada por Tepekuyu \& Topsakal (2015) com alunos de quinta série em uma escola em Istambul, Turquia, na qual sentimentos como medo, ódio, repugnância, dentre outros, foram associados aos insetos. Os autores apontaram, no entanto, que, embora as crianças entrevistadas tenham apresentado sentimentos negativos a respeito dos insetos, amor, amizade e bondade também foram apontados.

Características semióticas também foram relacionadas a esses animais, como, por exemplo, a crença de que alguns trazem sorte (22\%), como no caso do inseto esperança (Orthoptera), e de que, quando aparecem, indicam a chegada de chuva $(3 \%)$, como citado em relação às borboletas (Lepidoptera) e formigas (Hymenoptera). Crenças semióticas relacionadas a certos insetos estão presentes na sua relação com o homem desde a Antiguidade
(Cherry, 2006). Ulysséa (2010), em trabalho sobre a percepção e o uso de insetos por uma comunidade no interior de Santa Catarina, apresenta a crença dos entrevistados em superstições a respeito da previsão do tempo relacionadas ao canto matutino da cigarra e ao aumento do número de gafanhotos e libélulas em determinado local. Klein (2012) também aponta que certas comunidades relacionam sonhos envolvendo insetos a presságios, geralmente negativos.

\subsection{Usos diversos}

O uso de insetos e seus produtos pela comunidade estudada é restrito, estando relacionado principalmente ao caráter terapêutico (Tabela 3), com destaque para o mel de abelha (Hymenoptera), utilizado puro ou associado ao limão [Citrus limon (L.) Osbeck] no tratamento de enfermidades como gripe, pancadas e sinusite. $\mathrm{O}$ mel de mosquito (Hymenoptera), denominação dada a outro tipo de mel de abelha, que, segundo os informantes, é encontrado em estacas de madeira que compõem 
a formação das cercas, é utilizado principalmente para enfermidades que afetam a visão. Produtos à base de grilo (Orthoptera) foram apontados no tratamento de dor nas pernas e de ouvido. Como a comunidade classifica como insetos diversas espécies diferentes, foram citados, também, remédios à base de cobras (chás, banha e cachaça de cobra) - indicados em casos de envenenamento por picadas de cobras, tratamento de acidente vascular cerebral (AVC) e dor de ouvido - e à base de lacraia/escorpião (Scorpiones), raposa (Canidae) e gambá (Didelphidae), utilizados no tratamento de inflamações, hérnias e dores em geral. O uso de insetos no tratamento de enfermidades também é reportado por Ronghang et al. (2011) em estudo entre os karbis de Assam (Índia), no qual os insetos representam o segundo grupo de animais mais utilizado em tratamentos contra enfermidades na comunidade. São empregados integralmente, em partes ou em subprodutos. Quanto ao uso do mel de abelha no combate às doenças, Almeida (2010), Bagde (2014) e Melo et al. (2015) o referem para cura de conjuntivite, doenças do trato respiratório, gastrite, problemas na visão, diabetes, dentre ou-

TABELA 3 - Insetos* e produtos de insetos* utilizados no tratamento de enfermidades segundo moradores da comunidade Sussuapara, em Nazaré do Piauí (PI)

\begin{tabular}{|c|c|c|c|c|}
\hline $\begin{array}{c}\text { INSETOS/PRODUTO } \\
\text { DE INSETO }\end{array}$ & FORMA DE USO & INDICAÇÃO POPULAR & FL & ROP \\
\hline Mel de abelha & $\begin{array}{c}\text { Tomar puro ou em associação com } \\
\text { ervas ou limão ou aplicar em cima } \\
\text { do local afetado }\end{array}$ & $\begin{array}{c}\text { Gripe } \\
\text { Sinusite } \\
\text { Problemas de visão } \\
\text { Bronquite } \\
\text { Pancadas } \\
\text { Dor } \\
\text { Asma } \\
\text { Queimadura } \\
\text { Esporada de insetos venenosos } \\
\text { Câncer } \\
\text { Cisco no olho }\end{array}$ & 93,0 & 90,0 \\
\hline Mel de mosquito & Tomar puro & $\begin{array}{l}\text { Problemas de visão } \\
\text { Pancadas }\end{array}$ & 75,0 & 7,0 \\
\hline Grilo & $\begin{array}{l}\text { O entrevistado não soube informar } \\
\text { a forma de uso }\end{array}$ & $\begin{array}{l}\text { Dor de ouvido } \\
\text { Dor na perna }\end{array}$ & 33,0 & 10,0 \\
\hline Cobra & $\begin{array}{c}\text { Tintura: imergir na cachaça, deixar } \\
\text { descansar e tomar algumas doses; } \\
\text { infusão do chocalho; } \\
\text { in natura: usar a banha }\end{array}$ & $\begin{array}{c}\text { AVC } \\
\text { Dor de ouvido } \\
\text { Acidente envolvendo cobras peçonhentas }\end{array}$ & 45,0 & 50,0 \\
\hline Lacraia/Escorpião & $\begin{array}{l}\text { In natura: aplicar o intestino no } \\
\text { local }\end{array}$ & Inflamação & 100,0 & 20,0 \\
\hline $\begin{array}{l}\text { Raposa } \\
\end{array}$ & In natura: aplicar a banha no local & $\begin{array}{l}\text { Dor de ouvido } \\
\text { Hérnia } \\
\text { Dor na perna } \\
\text { Dor na coluna }\end{array}$ & 20,0 & 7,0 \\
\hline Gambá & In natura: aplicar a banha no local & Dor na perna & 100,0 & 10,0 \\
\hline
\end{tabular}

Legenda: FL: nível de fidelidade; ROP: prioridade de ordenamento. * Refere-se ao grupo de animais denominado de insetos, o qual abrange espécies pertencentes a diversos grupos externos à classe Insecta, mas que são reunidos em um único grupo de acordo com a comunidade Sussuapara. 
tros. O uso de escorpião/lacraia foi reportado em casos de dor de ouvido em levantamento de animais utilizados para fins medicinais realizado nas tribos Mawasi, na Índia (Bagde, 2014). A utilização de cobras também foi apontada por Odunta et al. (2012), em estudo sobre o uso de animais selvagens na terapia de enfermidades na Nigéria, e por Lima \& Santos (2010) como procedimento utilizado na medicina tradicional dos índios pancararu, no interior de Pernambuco. As cobras são indicadas no tratamento de ossos e articulações rompidas, dores nas costas, distúrbios da medula espinhal, reumatismo e inflamações. Lima \& Santos (2010) apresentam ainda informações sobre o uso de grilo como medicamento antidiurético e a utilização de raposa no combate ao reumatismo. Já Almeida (2010), em estudo sobre o uso de zooterápicos indicados na literatura médica clássica portuguesa e na zooterapia popular atualmente utilizada no Brasil, apresenta o uso de gambá no tratamento e prevenção de epilepsia.

As indicações sobre o uso terapêutico de insetos e seus subprodutos foram analisadas quanto ao grau de correspondência entre as respostas dos entrevistados de acordo com os usos citados e à distribuição do conhecimento entre os entrevistados. Para tanto, foram calculados o nível de fidelidade (FL) e a prioridade de ordenamento (ROP). Nesses índices, as citações que aparecem com resultado mais próximo a 100 são consideradas de maior relevância para a comunidade estudada, já que são utilizadas para um mesmo fim por um maior número de indivíduos. O uso terapêutico do mel de abelha (Hymenoptera), por exemplo, se mostrou como o mais relevante na comunidade, pois apresentou maior relação entre as indicações de uso e foi citado por um maior número de entrevistados, com $\mathrm{FL}=$ 93,0 e ROP = 90,0. O uso de escorpião (Scorpiones) e gambá (Didelphidae), ambos classificados como insetos pelos entrevistados, apresentou o índice máximo de correspondência sobre a forma de uso entre os entrevistados que os citaram $(F L=100)$. No entanto, não podem ser considerados como de maior relevância na comunidade, já que foram citados por um pequeno número de entrevistados, com ROP = 20 e $\mathrm{ROP}=10$, respectivamente.

A utilização de insetos na alimentação humana não foi relatada na comunidade, porém $7 \%$ informaram que algumas comunidades ao redor do mundo se alimentam de insetos, citando caranguejo $(3 \%)$, camarão $(2 \%)$, cobra $(1 \%)$ e gafanhoto $(1 \%)$. A etnocategoria inseto da comunidade Sussuapara abrange diversos táxons, além do Insecta. Nesse caso, embora não utilizem insetos em sua alimentação, alguns entrevistados citaram informações vistas em programas de televisão sobre o consumo de animais considerados por eles como insetos, em diversas outras populações. Foram percebidas reações de nojo por parte dos informantes ao se referirem a tais práticas alimentares.

Devido a sua localização em ambiente rural, acidentes com insetos peçonhentos são frequentes na comunidade. Foram reportados casos ocorridos com abelha (Hymenoptera) e marimbondo (Hymenoptera). Pelo fato de a comunidade classificar juntamente com insetos espécies pertencentes a outros grupos, a etnocategoria insetos peçonhentos abrange ainda: aranha (Araneae), lacraia/escorpião (Scorpiones), piolho-de-cobra (Scolopendromorpha), cobra (Squamata) e morcego (Chiroptera). O principal procedimento adotado em caso de acidentes provocados por tais animais é a ingestão de óleo de buriti (Mauritia flexuosa L. f.), utilizado em caso de acidentes com cobras. O encaminhamento do indivíduo ao serviço de saúde local só foi apontado para casos graves. A ingestão de cachaça de cobra após acidente com insetos peçonhentos também foi registrada. Nesses casos, a cachaça é armazenada 
juntamente com uma cobra morta e deixada em repouso por dias ou meses. A validade do produto varia de acordo com a deterioração da cobra. Outros procedimentos adotados após acidente com insetos peçonhentos são a utilização de mel de abelha, alho (Allium sativum L.), ingestão de leite, aplicação de álcool, uso de garapa de açúcar, aplicação de vinagre de cana-de-açúcar e aplicação de intestino de lacraia (Scorpiones) no local da picada. A utilização de óleo de buriti (M. flexuosa) no tratamento de envenenamento por cobra foi registrada por Matta (2010) no interior do Piauí. O uso de cachaça de cobra por comunidades após acidentes com cobras peçonhentas é apontado por Costa Neto (2000) e Barbosa et al. (2014). Em trabalho realizado na Paraíba, o uso de álcool, alho e lacraia é apontado no tratamento após acidentes com serpentes, abelhas, marimbondos e escorpião (Oliveira et al., 2013). Não foram encontrados relatos do uso de mel de abelha, leite, garapa de açúcar e vinagre de cana-de-açúcar após acidentes com insetos peçonhentos.

\subsection{Insetos na agricultura local e formas de combate}

Para os insetos considerados pragas pela comunidade, foi calculado o valor de importância da espécie $\left(\mathrm{IV}_{\mathrm{s}}\right)$. Nesse índice, as espécies com valor de $\mathrm{IV}_{\mathrm{s}}$ mais próximo a 1,0 apresentam maior importância local. Na comunidade Sussuapara, destacaram-se a lagarta (Lepidoptera) e a formiga (Hymenoptera) como espécies de maior importância local, com IV $=0,9 \mathrm{e} \mathrm{IV}_{\mathrm{s}}=0,7$, respectivamente, seguidas ainda pela pichilinga (Dermanyssus gallinae De Geer, 1778) e pelo cascudo (Hemiptera), ambos com IV $_{\mathrm{s}}=0,4$, e pelo gafanhoto (Orthoptera), com $\mathrm{IV}_{\mathrm{s}}=0,3$. As principais plantações atacadas por esses animais são as de arroz (O. sativa), feijão ( $V$. unguiculata, $P$. vulgaris, $P$. lunatus), milho (Z. mays) e mandioca (M. esculenta) (Tabela 4). A formiga (Hymenoptera) foi apontada como o inseto que ataca o maior número de plantações. A pichilinga (D. gallinae) foi a única espécie citada

TABELA 4 - Insetos pragas segundo moradores da comunidade Sussuapara, em Nazaré do Piauí (PI)

\begin{tabular}{|c|c|c|c|}
\hline INSETO* & ORGANISMO AFETADO & $\begin{array}{l}\text { NÚMERO DE } \\
\text { CITAÇÕES }\end{array}$ & $I V_{s}$ \\
\hline Lagarta & $\begin{array}{l}\text { Milho, feijão, arroz, mandioca, coentro, cana-de-açúcar, capim, } \\
\text { tomate, pimentão, melancia, cebola, banana }\end{array}$ & 77 & 0,9 \\
\hline Formiga & $\begin{array}{l}\text { Milho, feijão, arroz, mandioca, coentro, cebolinha-verde, cebola, } \\
\text { canafístula, berinjela, alface, laranja, seriguela, melancia, pimentão }\end{array}$ & 63 & 0,7 \\
\hline Cascudo & Feijão, arroz, mandioca, pimentão, melancia & 39 & 0,4 \\
\hline Mosca-branca & Feijão, arroz & 8 & 0,09 \\
\hline Minhoca & Coentro, pimentão & 5 & 0,05 \\
\hline Gorgulho & Milho, feijão, arroz & 5 & 0,05 \\
\hline Camaleão & Milho, feijão, arroz, mandioca, melancia, coentro & 4 & 0,04 \\
\hline Ganguji & Milho, coentro, cebolinha, alface & 4 & 0,04 \\
\hline Abelha & Arroz & 1 & 0,01 \\
\hline
\end{tabular}

Legenda: $\mathrm{IV}_{\mathrm{s}:}$ valor de importância da espécie. * Espécies apontadas como insetos pelos entrevistados. 
que ataca animais, sendo apontada como praga de galinha (Galliformes). Outros insetos também foram apontados como pragas, apresentando, no entanto, impacto local menos significativo (Tabela 4). Segundo Gullan \& Cranston (2012, p. 316), o "status de praga" está relacionado, dentre outros fatores, ao dano que os insetos podem causar em seus hospedeiros, bem como às altas densidades que esses animais podem atingir, fato percebido na comunidade, já que a percepção de insetos como pragas pelos entrevistados se deve ao fato de esses animais trazerem prejuízos econômicos na agricultura, bem como causarem incomodo ao invadir casas. Em estudo realizado em Cuiabá, Mato Grosso, os entrevistados associaram a pragas insetos que invadiam residências, como barata, formiga e mosquito, chamados de pragas urbanas, e não apontaram nenhuma espécie de inseto que atacasse plantações (Jorge et al., 2014). Tal fato é contrário ao que ocorreu no presente trabalho, em que a população informou como pragas apenas insetos que traziam prejuízos à lavoura. Esse resultado pode estar atrelado à localização rural da comunidade Sussuapara e à atividade de agricultura desenvolvida por quase todas as famílias entrevistadas. Resultados semelhantes foram encontrados por Alencar et al. (2012) em trabalho com comunidades rurais da Paraíba, em que foram consideradas pragas apenas insetos que traziam danos à agricultura. Foram apontadas, dentre outras, a formiga, a lagarta e a mosca-branca.

Com o intuito de evitar prejuízos econômicos causados pela destruição das plantações por insetos, a utilização de agrotóxicos é prática comum na comunidade, sendo adotado em todos os plantios. $\mathrm{O}$ uso de inseticidas químicos também foi registrado no combate a insetos presentes nas casas da comunidade. Para Montenegro et al. (2015), a utilização intensa de agrotóxicos se deve ao fácil acesso a tais produtos, à falta de conhecimento a respeito dos prejuízos ao meio ambiente e à saúde que esses produtos causam, ou, ainda, ao desconhecimento de formas naturais de combate a esses insetos. No caso da comunidade Sussuapara, é perceptível o fácil acesso a inseticidas sintéticos, bem como a crença de que o uso desses produtos é mais eficiente em comparação a outras formas de controle de pragas. Foi notado também que a maioria dos entrevistados $(60 \%)$ não conhece formas naturais de combate a insetos. Bioinseticidas foram citados por $40 \%$ dos informantes, que apontaram 14 espécies vegetais utilizadas apenas no controle de insetos em residências, sendo elas: alecrim (Lippia origanoides Kunth), com $66 \%$ das citações, seguido por bamburral (Hyptis suaveolens Poit.), alfavaca (Ocimum gratissimum L.), manjericão (Ocimum basilicum L.), carnaúba [Copernicia prunifera (Mill.) H. E. Moore], nim (Azadirachta indica A. Juss.), fumo (Nicotiana tabacum L.), arruda (Ruta graveolens L.), alho (Allium sativum L.), muçambê [Tarenaya spinosa (Jacq.) Raf.], angico-verdadeiro [Anadenanthera macrocarpa (Benth.) Brenan], fedegoso [Senna occidentalis (L.) Link] e mocó [Luetzelburgia auriculata (Allemão) Ducke]. Além do conhecimento de plantas no combate a insetos, o uso de fumaça de esterco ou de chifre bovinos, fumaça de pneu, água quente e casca de ovo também foi registrado. De acordo com os moradores, o conhecimento acerca de inseticidas naturais foi adquirido durante a infância, quando o uso de formas naturais de controle de insetos era rotineiramente praticado por seus pais. No entanto, embora alguns moradores conheçam outras formas de controle de insetos, a prática de controle natural não foi relatada como rotineira em nenhuma residência ou plantação na comunidade. A utilização de inseticidas químicos foi apontada por $100 \%$ dos entrevistados. O fato de a comunidade utilizar apenas inseticidas químicos 
no combate a insetos diverge do apresentado por Valadares \& Pasa (2010), os quais demonstram que agricultores de comunidades rurais, além do uso de inseticidas sintéticos, geralmente, conhecem e praticam formas naturais de controle de pragas. Entretanto, a forma de transmissão do conhecimento a respeito de bioinseticidas apresentada por uma parcela da comunidade Sussuapara é semelhante à apontada pelos autores acima, os quais afirmam que a difusão de tal conhecimento é considerada prática cultural, passada de pai para filho.

\section{Considerações finais}

A comunidade Sussuapara reconhece os insetos, atribuindo-lhes características tanto positivas como negativas. No entanto, o uso exclusivo de inseticidas sintéticos no combate a eles em suas plantações e residências, sem levar em consideração os danos causados por tais práticas, torna indispensáveis ações de educação ambiental com o intuito de sensibilizar a comunidade sobre os riscos do uso indiscriminado de agrotóxicos, bem como sobre a importância da utilização de práticas naturais no combate a insetos.

Por fim, a realização de trabalho relacionado ao conhecimento acerca dos insetos e de suas formas naturais de controle despertou, na comunidade, o interesse em relação à importância desses animais para a natureza e do uso de formas naturais de combate a insetos, possibilitando, dessa forma, a realização de atividades locais de etnoconservação.

\section{Referências}

Aguiar, R. B.; Gomes, J. R. C. (Orgs). Projeto cadastro de fontes de abastecimento por água subterrânea, estado do Piauí: diagnóstico do município de Nazaré do Piauí. . Fortaleza: CPRM - Serviço Geológico do Brasil, 2004.

Alencar, J. B. R.; Silva, E. F.; Santos, V. M.; Soares, H. K. L.; Lucena, R. F. P.; Brito, C. H. Percepção e uso de "insetos" em duas comunidades rurais no semiárido do estado da Paraíba. Revista de Biologia e Farmácia, 9, 72-91, 2012. Disponível em: <http://sites.uepb.edu.br/biofar/download/v-especial-2012/PERCEP $\%$ C3\%87\%C3\%83O\%20E\%20USO $\% 20$ DE $\% 20 \%$ E2\%80\%9CINSETOS $\%$ E2\%80\%9D $\% 20$ EM $\% 20$ DUAS\%20COMUNIDADES\%20RURAIS\%20NO\%20 SEMI\%C3\%81RIDO\%20DO\%20ESTADO \%20DA\%20 PARA $\%$ C3\%8DBA.pdf $>$.

Almeida, A. V. Zooterapia usada na prevenção e tratamento da "gota coral" (epilepsia): passado e presente. Etnobiología, 8, 59-74, 2010. Disponível em: <http://www. asociacionetnobiologica.org. $\mathrm{mx} / \mathrm{mx} 2 /$ images/documents/ revista\%208/Argus\%20Vasconcelos.pdf $>$.

Almeida Neto, J. R.; Costa Neto, E. M.; Silva, P. R. R.; Barros, R. F. M. de. Percepções sobre insetos em duas comu- nidades rurais da Serra do Passa Tempo, Nordeste do Brasil. Espacios, 36(11), 1-12, 2015. Disponível em: <http://www. revistaespacios.com/a15v36n11/15361113.html>.

Alves, R. R. N.; Souto, W. M. S. Ethnozoology in Brazil: current status and perspectives. Journal of Ethnobiology and Ethnomedicine, 7 (22) 18 p., 2011. doi: 10.1186/17464269-7-22

Bagde, N. Indigenous knowledge of Zootherapeutic use of Invertebrate by the Mawasi tribes of Chhindwara District of Madhya Pradesh India. International Journal of Life Sciences, 2(3), 244-248, 2014. Disponível em: $<$ http://oaji. net/articles/2014/736-1413219939.pdf>.

Barbosa, A.; Oliveira, D. D. S. C.; Meira, C. R. Uso tradicional da fauna silvestre do município de Lapão - Bahia. Enciclopédia Biosfera, 10(18), 16, 2014. Disponível em: $<$ http://www.conhecer.org.br/enciclop/2014c/pdf/uso $\% 20$ tradicional.pdf $>$.

Begossi, A.; Lopes, P. F.; Oliveira, L.E. C.; Nakano, H. Ecologia de pescadores artesanais da Baía de Ilha Grande. IBIO/Ministério da Justiça. Apoio: Capesca: Preac/CIS- 
-Guanabara/Lepac/CMU [UNICAMP] \& IDRC, Canadá. Rio de Janeiro. 2009.

Byg, A.; Balslev, H. Diversity and use of palms in Zahamena, eastern Madagascar. Biodiversity and Conservation, 10, 951-970, 2001. doi: 10.1023/A:1016640713643

Cajaiba, R. L.; Silva, W. B. Percepção dos alunos do ensino fundamental sobre os insetos antes e após aulas práticas: um caso de estudo no município de Uruará - Pará, Brasil. Lugares de Educação, 5 (11), 118-132, 2015. Disponível em: <http://www.biblionline.ufpb.br/ojs2/index.php/rle/ article/download/19943/pdf>.

CEPRO. Fundação Centro de Pesquisas Econômicas e Sociais do Piauí. Diagnóstico Sócioeconômico. Município Nazaré do Piauí. 2010. Disponível em <http://www.cepro. pi.gov.br/download/201105/CEPRO03_ec1f27d548.pdf> Acesso em: ago. 2014.

Cherry, R. Insects in the choctaw emergency mythology. American Entomologist, 52(1), 20-22, 2006. Disponível em: $<$ http://ae.oxfordjournals.org/content/ae/52/1/20.full.pdf $>$.

Costa Neto, E. M. Conhecimento e usos tradicionais de recursos faunísticos por uma comunidade afro-brasileira. Resultados preliminares. Interciência, 25(9), 423-431, 2000. Disponível em: <http://www.redalyc.org/articulo. oa? $\mathrm{id}=33905105>$.

Costa Neto, E. M. Etnoentomologia do povoado de Pedra Branca, município de Santa Teresinha, Bahia. Um estudo de caso das interações seres humanos/insetos. Tese (Doutorado em Ecologia e Recursos Naturais) - UFSC, 2003.

Costa Neto, E. M.; Pacheco, J. M. A construção do domínio etnozoológico "inseto" pelos moradores do povoado de Pedra Branca, Santa Terezinha, Estado da Bahia. Acta Scientiarum, 26(1), 81-90, 2004. Disponível em: <http:// eduem.uem.br/ojs/index.php/ActaSciBiolSci/article/ view/1662/1004>.

Durst, P. B.; Shono, K. Edible forest insects: exploring new horizons and traditional practices. In: Durst, P. B.; Johnson, D. V.; Leslie, R. N.; Shono, K. (Orgs.). Forest insects as food: humans bite back. Proceedings of a workshop on Asia-Pacific resources and their potential for development. Thailand. 2010. p 1-4. Disponível em: <https://cisr.ucr.edu/ pdf/edible-forest-inescts.pdf $>$.

Ferreira, M. N.; Ballester, W. C.; Dorval, A.; Costa, R. B. Conhecimento tradicional dos Kaiabi sobre abelhas sem ferrão no Parque Indígena do Xingu, Mato Grosso, Brasil. Tellus, 19, 129-144, 2010. Disponível em: <http://www. tellus.ucdb.br/index.php/tellus/article/download/212/250>.

Friedman, J.; Yaniv, Z.; Dafni, A.; Palewith, D. A preliminar classification of the healing potencial of medicinal plants, based on a rational analysis of an ethnopharmacological field survey among bedouins in the Negev desert, Israel. Journal of Ethnopharmacology, 16, 275-287, 1986. doi:10.1016/0378-8741(86)90094-2

Gullan, P. J.; Cranston, P.S. Os insetos: um resumo de entomologia. 4. ed. São Paulo: Roca, 2012.

Huberman, A. M.; Miles M. Analyse des données qualitatives: recueil de nouvelles méthodes. De Boeck Université, 1991.

ITIS - Integrated Taxonomic Information System. 2015. Disponível em: <http://www.itis.gov/>. Acesso em: nov. 2015.

Jorge, V. C.; Sánchez, D. C. M.; Souza, M. D.; Pasa, M. C.; Rezende, E. H. Conhecimentos entomológicos pelos moradores do bairro Verdão em Cuiabá - MT. Enciclopédia Biosfera, 10(18), 3814-3824. 2014. Disponível em: <http:// www.conhecer.org.br/enciclop/2014a/MULTIDISCIPLINAR/Conhecimentos.pdf $>$.

Kellert, S. R. Values and perceptions of invertebrates. Conservation Biology, 7(4), 845-855, 1993. doi: 10.1046/j.15231739.1993.740845.x

Klein, B. A. The curious connection between insects and dreams. Insects. 3, 1-17, 2012. doi: 10.3390/insects3010001

Lima, J. R. B.; Santos, C. A. B. Recursos animais utilizados na medicina tradicional dos índios Pankararu no Nordeste do estado de Pernambuco, Brasil. Etnobiología, 8, 39-50, 2010. Disponível em: $<\mathrm{http}$ ://asociacionetnobiologica.org. $\mathrm{mx} /$ aem/wp-content/uploads/Revista-etnobiologia-8.pdf $>$.

Lopes, L. A.; Valduga, M.; Athaydes, Y.; Dal-Farra, R. A. As concepções sobre insetos no ensino fundamental em escola pública de Sapucaia do Sul, RS. Acta Scientiae, 16(4), 214223, 2014. Disponível em: <http://www.periodicos.ulbra. br/index.php/acta/article/viewFile/1276/1028>.

Mahawar, M. M; Jaroli, D. P. Traditional zootherapeutic studies in India: a review. Journal of Ethnobiology and Ethnomedicine, 4(17), 12, 2008. doi: 10.1186/1746-4269-4-17

Matta, P. Conhecimento tradicional, biodiversidade e repartição de benefícios: o caso dos produtores de óleo 
de buriti de Palmeira do Piauí. CAMPOS - Revista de Antropologia Social, 11(2), 113-132, 2010. doi: 10.5380/ cam.v11i2.21047

Melo, A. C. B.; Araújo, F. L.; Freire, J. E.; Braga, P. E. T. O conhecimento popular acerca dos insetos no município de Cariré, Ceará, Brasil. Ciência e Natura, 37(2), 253-260, 2015. Disponível em: $<$ http://cascavel.ufsm.br/revistas/ojs2.2.2/index.php/cienciaenatura/article/download/14997/ pdf $>$.

Meyer-Rochow, V. B. Entomophagy and its impact on world cultures: the need for a multidisciplinary approach. In: Durst, P. B.; Johnson, D. V.; Leslie, R. N.; Shono, K. (Orgs.). Forest insects as food: humans bite back. Proceedings of a workshop on Asia-Pacific resources and their potential for development. Thailand. 2010. p. 23-36. Disponível em: $<$ http://foodfactoryfoundation.org/documents/Edible\%20 insects.pdf $>$.

Meyer-Rochow, V. B; Chakravorty, J. Notes on entomophagy and entomotherapy generally and information on the situation in India in particular. Applied Entomology and Zoology, 48, 105-112, 2013. doi: 10.1007/s13355013-0171-9

Modro, A. F. H.; Souza, S.; Aburaya, F. H.; Maia, E. Conhecimento dos moradores do médio Araguaia, Estado do Mato Grosso, sobre a utilidade de produtos de abelhas (Hymenoptera, Apidae). Acta Scientiarum, 31(4), 421-424, 2009 a.

Modro, A. F. H.; Costa, M. S.; Maia, E.; Aburaya, F. H. Percepção entomológica por docentes e discentes do município de Santa Cruz do Xingu, Mato Grosso, Brasil. Biotemas, 22(2), 153-159, 2009b. doi: 10.5007/2175-7925.2009v22n2p153

Montenegro, Í. F.; Alencar, J. B. R.; Silva, E. F.; Lucena, R. F. P.; Brito, C. H. Conhecimento, percepção e uso de animais categorizados como "insetos" em uma comunidade rural no semiárido do estado da Paraíba, Nordeste do Brasil. Gaia Scientia. 8(2), 250-270, 2015. Disponível em: <http://periodicos.ufpb.br/ojs2/index.php/gaia/article/ download/22712/12589>.

Mucchielli, A. Dictionnaire des méthodes qualitatives en sciences humaines et sociales. Paris: Armand Colin. 1996.

Oduntan, O. O.; Akinyemi, A.; Ojo, O.; Ogunyode, O.; Adesina, O. Survey of Wild Animals Used in Zoo-Therapy at Ibadan, Oyo State, Nigeria. International Journal of
Molecular Zoology, 2(9), 70-73, 2012. doi: 10.5376/ ijmz.2012.02.0009

Oliveira, H. F. A.; Costa, C. F.; Sassi, R. Relatos de acidentes por animais peçonhentos e medicina popular em agricultores de Cuité, região do Curimataú, Paraíba, Brasil. Revista Brasileira de Epidemiologia, 16(3), 633-643, 2013. doi: 10.1590/S1415-790X2013000300008

Petiza, S.; Hamada, N.; Bruno, A. C.; Costa Neto, E. M. Etnoentomología baniwa. Boletín de la Sociedad Entomológica Aragonesa, 52, 323-343, $2013 \mathrm{a}$.

Petiza, S.; Hamada, N.; Bruno, A. C.; Costa Neto, E. M. Etnotaxonomia entomológica baniwa na cidade de São Gabriel da Cachoeira, estado do Amazonas, Brasil. Revista de Antropologia, 5(3), 708-732, 2013b. Disponível em: $<$ http://periodicos.ufpa.br/index.php/amazonica/article/ viewFile/1602/2001>.

Ramandey, E; Mastrigt, H. V. Edible insects in Papua, Indonesia: from delicious snack to basic need. In: Durst, P. B.; Johnson, D. V.; Leslie, R. N.; Shono, K. (Orgs.). Forest insects as food: humans bite back. Proceedings of a workshop on Asia-Pacific resources and their potential for development. Thailand. 2010. p 103-114. Disponível em: $<$ http://www.fao.org.webtranslate-widget.systransoft.com/ docrep/012/i1380e/i1380e00.pdf>.

Rocha, M. S. P.; Mourão, J. S.; Souto, W. M. S.; Barboza, R. R. D.; Alves, R. R. N. O Uso dos recursos pesqueiros no estuário do Rio Mamanguape, estado da Paraíba, Brasil. Interciência, 3(12), 903-909, 2008. Disponível em: <http:// www.interciencia.org/v33_12/903.pdf>.

Ronghang, R.; Teron, R.; Tamuli, A. K.; Rajkhowa, R. C. Traditional zootherapy practised among the karbis of Assam (India). The Ecoscan, 1, 161-166, 2011. Disponível em: <http://theecoscan.in/journalpdf/spl2011_v1-27\%20 ronjit\%20ronghang.pdf $>$.

Souza Junior, A. A.; Costa Neto, E. M.; Santos, G. C. B. As concepções que estudantes da sexta série do ensino fundamental do Centro de Educação Básica da Universidade Estadual de Feira de Santana possuem sobre os insetos. Gaia Scientia, 8(1), 8-16, 2014. Disponível em: <http:// www.ies.ufpb.br/ojs2/index.php/gaia/article/viewFile/17924/10217>.

Souza Júnior, J. R.; Lima, É. F. B. Representações locais sobre insetos em hortas comunitárias e mercados públicos da cidade de Teresina, Piauí. In: Costa Neto, E. M. (Org.). 
Antropoentomofagia, insetos na alimentação humana. Feira de Santana: UEFS Editora, 2014. p. 607-620.

Tepekuyu, H. Z.; Topsakal, Ü. U. Turkish Primary School Students' Attitudes toward 'Disgusting Animals': Insects. International Journal for Innovation Education and Research, 3, 15-25, 2015. Disponível em: <http://www. ijier.net/assets/turkish-primary-school-students-ijier.netvol-3-3-2.pdf>.

Trindade, O. S. N.; Silva Júnior, J. C.; Teixeira, P. M. M. Um estudo das representações sociais de estudantes do ensino médio sobre os insetos. Ensaio, 14(3), 37-50, 2012. Disponível em: $<$ http://www.portal.fae.ufmg.br/seer/index. php/ensaio/article/viewFile/458/1076>.
Ulysséa, M. A.; Hanazaki, N.; Lopes, B. C. Percepção e uso dos insetos pelos moradores da comunidade do Ribeirão da Ilha, Santa Catarina, Brasil. Biotemas, 23, 191-202, 2010. doi: 10.5007/2175-7925.2010v23n3p191

Valadares, L. C. A.; Pasa, M. C. Pest control methods used by riverine from Rio Vermelho community, South of Mato Grosso State, Brazil. Biodiversidade, 9(1), 4-14, 2010. Disponível em: <http://200.129.241.78/index.php/ biodiversidade/article/viewFile/97/88>.

Wester, L. Yongvanit, S. Naming consistency for forest plants in some rural communities of Northeast Thailand. Ethnobotany Research \& Applications, 4, 203-212, 2006. 